\title{
Assessment of Dentists' Knowledge Concerning the Management of Pregnant Women in the Dental Office
}

\author{
Ilea $A^{1}$, Lazăr $A C^{1}$, Morar $A E^{2}$, Boșca $A B^{* 3}$, Băbțan $A M^{1}$, Petrescu $\mathrm{NB}^{1}$, Uriciuc $\mathrm{WA}^{1}$, Feurdean $\mathrm{CN}^{1}$, \\ Câmpian RS ${ }^{1}$ and Șovrea $A^{3}$ \\ ${ }^{1}$ Department of Oral Rehabilitation, Oral Health and Dental Office Management, Faculty of Dentistry, "Iuliu Hațieganu” University of \\ Medicine and Pharmacy, Cluj-Napoca, Romania \\ ${ }^{2}$ Postgraduate student of Faculty of Dentistry, "Iuliu Hațieganu" University of Medicine and Pharmacy, Cluj-Napoca, Romania \\ ${ }^{3}$ Department of Histology, Faculty of Medicine, "Iuliu Hațieganu” University of Medicine and Pharmacy, Cluj-Napoca, Romania
}

*Corresponding author: Lecturer Bianca Adina Boșca, Department of Histology, Faculty of Medicine, "Iuliu Haţieganu” University

of Medicine Cluj-Napoca, Str. L. Pasteur, No. 4, Cluj-Napoca, Romania

\section{ARTICLE INFO}

Received: 彗 February 11, 2019

Published: 蔧 February 26, 2019

Citation: Ilea A, Lazăr AC, Morar AE, Boșca AB, Băbțan AM, et al. Assessment of Dentists' Knowledge Concerning the Management of Pregnant Women in the Dental Office. Biomed J Sci \& Tech Res 15(2)-2019. BJSTR. MS.ID.002667.

Keywords: Pregnancy; Dental Treatment; Dentistry; Dental Procedures; Medical Education

\section{ABSTRACT}

Introduction: Pregnancy is a physiological condition characterized by multiple changes in the entire woman's body, including the oral cavity. Gingival hyperplasia, gingivitis, pyogenic granulomas and some salivary changes are common conditions in pregnant women.

Purpose: To assess the dental practitioner's medical knowledge regarding the dental maneuvers allowed during pregnancy and the drug prescriptions for these patients in the dental office.

Methodology: In the present study, 128 active dentists with professional experience of one to 30 years were included. An online questionnaire was applied using the www. isondaje.ro platform. The questionnaire was intended to be anonymous, with responses being collected online. The first set of questions assessed the socio-demographic data, the specialization, and the professional experience. The second set of questions referred to the therapeutic approach of the pregnant women: the type of treatment and the period during pregnancy when they chose to perform the dental treatments. The third set of questions referred to the dental X-rays recommendation in pregnant women. The last category of questions addressed to the medication prescribed or administered to pregnant patients.

Results: The data were quantified by calculating the arithmetic averages. Most participants had experience in the medical dental practice, therefore the answers were relevant for this study. More than half of the doctors surveyed stated that they would only treat a pregnant woman in emergency situations. Less than half would treat a pulp pathology only in emergency, but a pulp gangrene at any time during pregnancy. Regarding the extractions, the majority of the dentists would prefer to delay the treatment.

Conclusion: An important number of dentists have insufficient knowledge related to the dental treatments that can be performed in a pregnant woman. Therefore, post-graduate courses are required in order to up-date the knowledge of dentists.

\section{Introduction}

Pregnancy is a physiological condition characterized by multiple changes in the entire the woman's body, including the oral cavity. Maintaining the dental and oral health during pregnancy is crucial, since there are specific limitations in the treatment of several oral diseases.

\section{Systemic Physiological Adaptations in the Body During Pregnancy}

The systemic changes that occur in pregnancy encompass various anatomical and physiological adaptations in order to nurture 
and to accommodate the developing fetus. These changes begin after the conception and affect every system in the body such as:

a. Increased number of blood cells and plasma volume

b. Increased level of coagulation factors

c. Increased fibrynolitic activity

d. Iron deficiency and anemia

e. Tachycardia

f. Increased cardiac output

g. Increased heart rate

h. Movement of the diafragm to the top

i. Increased risk of apneea and dyspneea

j. Hyperventilation

k. Nausea and vomiting

l. Stomach acid burns

m. Behavioral changes

n. Increased nutritional needs [1].

\section{Decompensation of Different Organs}

During pregnancy, the normal function of various organs can be impaired, resulting in pathological manifestations that could interfere with the oral therapeutic interventions.

Gestational Diabetes Mellitus (GDM): Is defined as hyperglycemia that occurs for the first time during pregnancy. This may include cases of type 2 diabetes that have not been previously diagnosed but became symptomatic in early pregnancy, and true GDM which occurs later. GDM affects between 2 and 5\% of pregnant women. Untreated, GDM could induce, besides the general complications, oral diseases, such as gingivitis and periodontal disease [2].

Arterial Hypertension: is one of the most common medical disorders in pregnancy and could lead to maternal fetal complications. The dentist should collaborate with the cardiologist or the gynecologist before performing any dental treatment if the pregnant woman suffers from hypertension, especially severe hypertension, and is under anti-hypertensive medication $[2,3]$.

Conditions Requiring Anticoagulant Therapy: If heparin is administered to the pregnant patient and a bleeding oral intervention is necessary, the dentist should ask the physician to adjust the dose of anticoagulant in order to ensure an INR (International Normalised Ratio) value lower than 2.2. [2,4].

\section{Drug Administration to the Pregnant Women}

Since most medicines have side effects, these should be avoided during pregnancy and administered only in serious conditions. Thus, the use of medication is not recommended in the first trimester, especially during the first 13 weeks of gestation. According to the potential adverse effects and the risk to the fetus $(1,6,7)$, US Food and Drug Administration (FDA) classified drugs into five categories:

A. A - Human studies showed no risk to the fetus,

B. B - Animal studies reported no fetal risk; there are no studies in pregnant women; E.g: Amoxicillin, Cephalexin, Chlorhexidine, Clindamycin, Erythromycin, Metronidazole, Penicillin, Acetaminophen, Ibuprophen, Lidocaine, Prilocaine, Pilocarpine, Prednisolone,

C. C - Animal studies reported a risk to the fetus; no studies have been performed on pregnant women; E.g: Ciprofloxacin, Clarithromycin, Codeine with acetaminophen, Hydrocodone with acetaminophen, Bupivacaine, Epinephrine, Mepivacaine;

D. D - These drugs should only be used in exceptional cases; E.g: Gentamicin, Doxycycline, Tetracycline;

E. $\quad \mathrm{X}$ - Risks of using these drugs in pregnant women are much higher than benefits [5-7].

Analgesics: The most common analgesic used during pregnancy is acetaminophen (Paracetamol). This is the safest, being included in category B by the FDA. The most commonly reported side effect of paracetamol is hepatotoxicity [1,8]. Other analgesics, such as ibuprophen, are also included in category B, but only in the first and second trimesters. In the third trimester, it falls into category D because it decreases the amount of amniotic fluid, and also limits the vaginal dilation during labor $[1,9]$.

Antibiotics: Most of the antibiotics recommended by the dentists are classified in category B, except for gentamicin, doxycycline and tetracycline, which are included in category

Gentamicin has been Reported to Cause Fetal Toxicity: Whereas doxycycline and tetracycline cause dental dyschromia and have a detrimental effect on bone development. Ciprofloxacin, a broad-spectrum antibiotic, is commonly prescribed in periodontal diseases associated with Actinobacillus infection. Recent evidence suggest that Ciprofloxacin is involved in arthropathy and has severe effects on cartilage developing, therefore it is not recommended for pregnant women [10]. Metronidazole, included in group B, is banned in the first trimester because it has teratogenic effects [11].

\section{Oral Manifestations During Pregnancy}

Oral manifestations during pregnancy include gingival hyperplasia, gingivitis, pyogenic granulomas and some salivary changes. Oral health should be closely assessed and monitored both before and during pregnancy. However, if the exacerbation of an oral disease occurs during pregnancy, the dentist should be aware that dental treatments are not completely contraindicated. The most appropriate time to perform dental treatments is the second trimester of pregnancy. The dental treatments in the first trimester of pregnancy is influenced by manifestations such as: accentuated 
vomiting reflex, drowsiness and fatigue. In the third trimester, only emergency and short interventions are recommended [2,5,12].

Gingivitis: Gingivitis is an acute and superficial inflammation of the marginal gingiva and interdental papilla, that occurs mostly in the second or third trimester of pregnancy. Local signs include: erythema, hyperplasia, increased amount of gingival sulcular fluid, increased volume of the marginal gingiva and interdental papillae, with the formation of false periodontal pockets. The anterior part of the dental arches is the most affected, but gingivitis can be localized in a group of teeth or even generalized in the entire oral cavity $[13,14]$. The local examination usually reveals elevated bacterial plaque counts, gingival bleeding, and false periodontal pockets with epithelial insertion still maintained, and no alveolar bone resorption. After birth, these changes usually disappear [4].

The Pregnancy Epulis: The pregnancy epulis is a tumorlike lesion situated on the gingiva, but may also develop on the tongue, lips, jugular mucosa, or palate [13]. It is less common than gestational gingivitis and occurs in the second or third month of pregnancy. The epulis is the result of an increased inflammatory response to the dental plaque and other irritants. Favorable factors are: poor oral hygiene, presence of tartar and high levels of progesterone in the gingival tissues [15]. The lesion is soft upon palpation, grows rapidly and bleeds easily $[4,13]$. The pregnancy epulis generally regresses in up to 3 months after birth. Surgical removal of the epulis is recommended if the lesion does not regress after this interval [4].

Acute Pulpitis: Acute pulpitis should be prevented by prophylaxis and regular check-ups. The correct treatment consists in vital pulpectomy under plexal anesthesia without a vasoconstrictor, administered by gentle maneuvers [5]. Devital pulp extirpation is contraindicated because the diffusion of the devitalizing substances beyond the root apex which cannot be controlled. However, if such a procedure is required, an arsenic-free devitalizer is recommended [5].

Pulp Necrosis: Pulp necrosis is difficult to manage during pregnancy. Asymptomatic teeth with necrotic pulp affected before pregnancy should be extracted. Several cases of repetitive spontaneous abortions have been reported in patients due to dental infections [5].

\section{Bleeding Dental Treatments and Imagistic Investigation in Pregnant Women}

Dental Extraction: Extractions can be performed after the 4th month and before the end of the 8th month of pregnancy. The extraction is recommended to remove only the symptomatic teeth that cannot be recovered but could generate local suppurations [5].

Dental Radiography in Pregnant Women: are contraindicated throughout pregnancy by some authors. Radiographs are strictly contraindicated in the first 8 months of pregnancy, and especially during the first trimester. In exceptional cases, the radiographs can be performed only using appropriate protection. In the 9th month of gestation, the risk for fetus is much reduced [5]. The National Commission for Radiation Protection argues that fetal exposure at $50 \mathrm{mSv}$ will not cause congenital defects, and if a radiological examination is essential for establishing the diagnosis, it is allowed [16]. The opportune moment to perform an X-ray during pregnancy is the second trimester [17].

\section{Aim}

The aim of our study was to assess dental practitioners' knowledge regarding the drug prescription and the particularities of the dental therapeutic procedures performed during pregnancy.

\section{Material and Methods}

In this study, we assessed the knowledge of dental practitioners regarding the management of pregnant women in the dental office by distributing an online questionnaire using the www.isondaje. ro platform. The study was attended by 128 dentists, of various specialties, aged between 24 and 55 years, of which 123 practiced in the urban area, and 5 in the rural area. The questionnaire was intended to be anonymous, with the responses collected online. The time for completing the questionnaire was unlimited, with an average duration between three and four minutes, depending on the answers chosen, including mandatory and optional questions. The first set of questions (1 to 3 ) referred to the socio-demographic data including the medical specialty. The second set of questions (4 to 9) referred to the therapeutic approach of the pregnant women: the medical procedures they perform and what period during pregnancy they chose for the dental treatments. These questions had five variants of choice, with the possibility of one or multiple choices. The answer options were:

a. Only in case of emergency,

b. Only in the first trimester,

c. Only in the second trimester of pregnancy,

d. Only in the third trimester and

e. At any time during pregnancy.

The tenth question referred to the dental radiographs recommendation in pregnant women. This question had five variants of choice, with the possibility of several options:

a. I do not want to be responsible for some complications,

b. Only in the first trimester,

c. Only in the second trimester,

d. Only in the third trimester and

e. Yes, at any time during pregnancy.

The last set of questions (11 and 12) addressed to the administration of the anesthetic substance and the type of medication used or prescribed in pregnant patients. These questions had two 
variants of response: (a) no and (b) yes, and it was followed by an open question regarding the most frequently prescribed anesthetic or antibiotic, respectively. The statistical processing of the data employed Microsoft Excel, and the results were presented by descriptive statistics.

\section{Results}

\section{Dentists' Distribution According to their Age}

Out of the 128 subjects, six dentists (5\%) were under 25 years of age, 85 dentists (66\%) were aged between 26 and 35 years, 32 dentists (25\%) were aged between 36 and 45 years, and 5 dentists were aged between 46 and 55 years (4\%). The group consisting of dentists aged between 26 and 35 years, was the largest, followed by the group of dentists aged between 36 and 45 years. These results can be explained by the experience of the young doctors in using the online environment.

\section{Dentists' Distribution According to the Medical Specialty}

The participants in the study included: 97 specialists in general dentistry (75.7\%), 11 dental practitioners specialized in dental prosthetics (8.59\%), 8 dento-alveolar surgeons (6.25\%), 7 specialists in endodontics (5.46\%), 3 orthodontists (2.34\%) and 2 specialists in periodontics (1.56\%). Therefore, the best represented specialty was general dentistry.

\section{The Therapeutic Approach and the Medical Procedures Performed in Pregnant Women}

To the question regarding their attitude during the anamnesis when they are informed that the patient is pregnant, none of the dentists would refuse to treat her (the first option), 2 dentists $(1.6 \%)$ would send the patient to the specialized medical services in case of emergency (the second option), 86 doctors (67.2\%) chose to perform only procedures that do not require anesthesia (the third option), and 40 doctors (31.2\%) would treat a pregnant woman like any other patient (the last option). Thus, the third approach was chosen by most of the dentists. One third of the attending physicians would treat the pregnant woman as if she was a regular patient, without considering any of the particular aspects or risks regarding this condition (Figure 1).

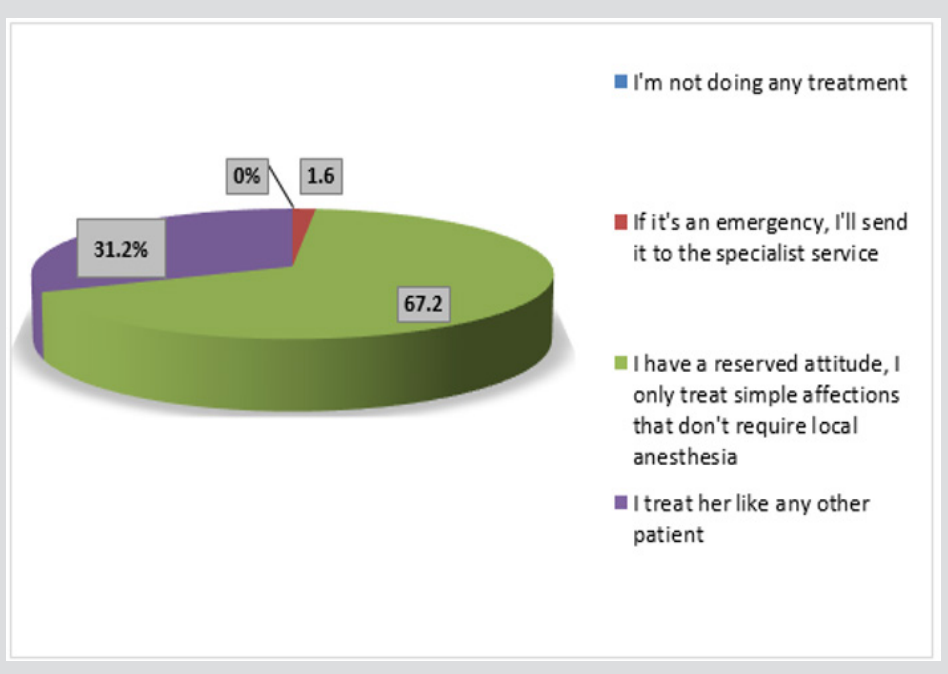

Figure 1: Graphical representation of the dentists' attitude regarding the therapeutic approach of a pregnant woman.

\section{Dental Treatments in the Pregnant Patient}

The fourth question referred to the period during pregnancy when a dental treatment can be performed had five variants of choice, with the possibility of one or multiple choices. The answer options were:
a. Only in case of emergency,
b. Only in the first trimester,
c. Only in the second trimester of pregnancy,
d. Only in the third trimester and
e. At any time during pregnancy.

Since it was a question with several possible choices, there were 170 answers, of which 55 answers (32.4\%) were the first option a. Only three answers (1.8\%) were option

b. $\quad 41$ answers $(24.1 \%)$ were option three

c. 8 answers $(4.7 \%)$ were option and

d. 63 answers (37\%) were the last option

e. The majority of the answers were the two opposite options: would perform the dental treatment at any time or would treat pregnant patients only in case of emergency.

\section{Endodontic Treatment of Pulp Diseases in the Pregnant Patient}

The fifth question referred to the period during pregnancy when the endodontic treatment can be performed. Since it was a question with several possible choices, there were 172 responses, among which: 

a. 74 answers (43\%) were the first option,
b. Only 4 answers $(2.3 \%)$ were the second option,
c. 45 answers $(26.2 \%)$ were the third option,

d. 10 answers (5.8\%) were the fourth variant and

e. 39 answers $(22.7 \%)$ were the last option (Figure 2).

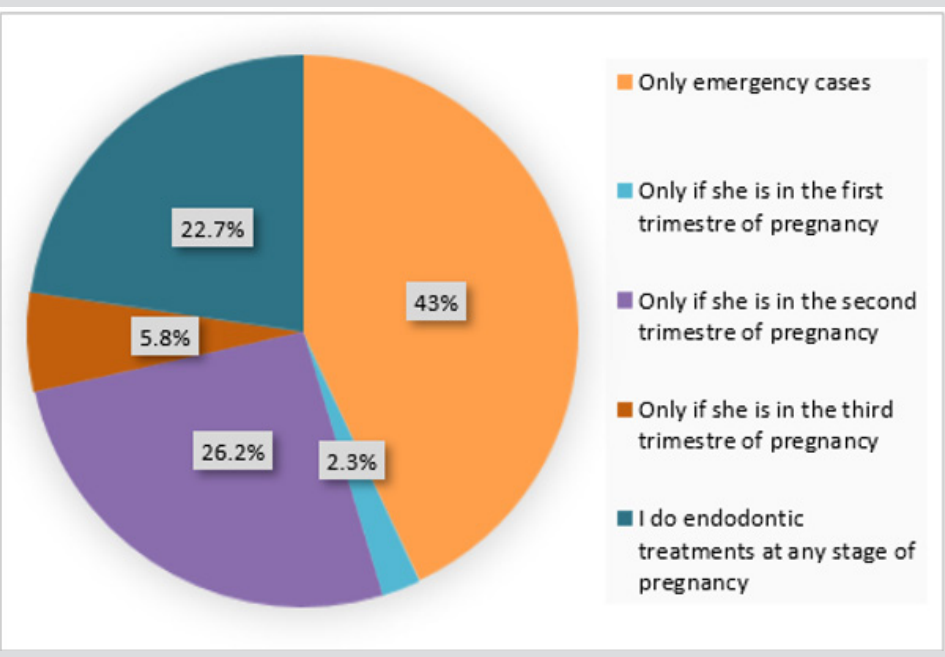

Figure 2: Graphical representation of the appropriate period for the endodontic treatments performed in pregnant patients.

\section{Endodontic Treatment of the Necrotic Pulp in the Pregnant Patient}

The sixth question referred to the period during pregnancy when the endodontic treatment for pulp necrosis can be performed. There were 164 answers, of which:

a. 55 answers (33.5\%) were the first option, b. Only 2 answers (1.2\%) were the second option,

c. 29 answers $(17.7 \%)$ were the third option,

d. 7 answers (4.3\%) were the fourth variant and

e. $\quad 71$ answers (43.3\%) were the last option.

The last option collected the majority of the answers, followed by the first variant (Figure 3).

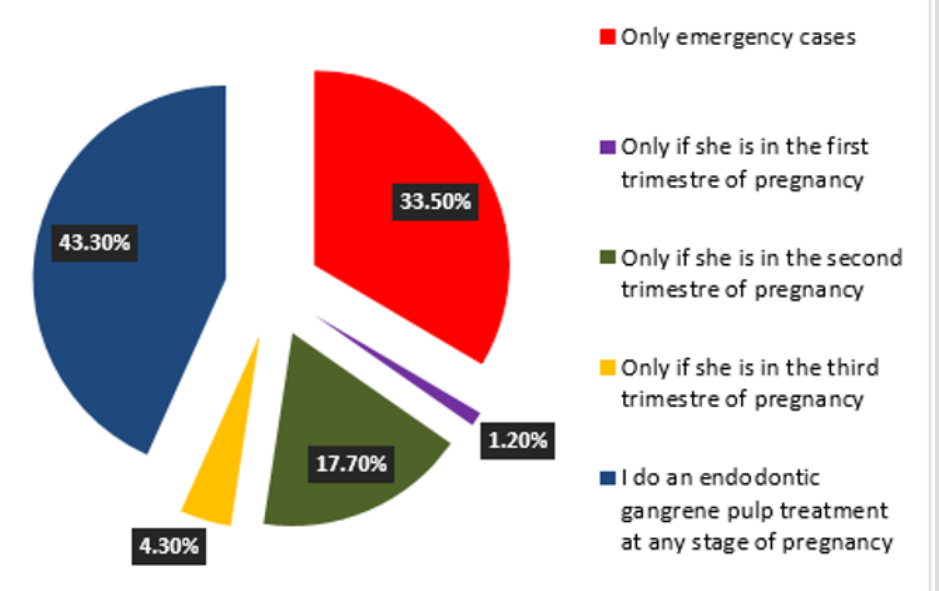

Figure 3: Graphical representation of the appropriate period for the endodontic treatment of the pulp necrosis performed in pregnant patients.

\section{Specific Treatment for Gingivitis in the Pregnant Patient}

The seventh question referred to the period during pregnancy when the treatment for gingivitis can be performed.

a. There were 138 answers, of which 21 answers (15.2\%) were the first option, b. Only 4 answers (2.9\%) were the second option,

c. 12 answers $(8.7 \%)$ were the third option,

d. 4 answers $(2.9 \%)$ were the fourth variant and

e. 97 answers (70.3\%) were the last option. 
The last option was chosen by the majority of the dentists, showing that most subjects were well informed about gingivitisspecific treatment. A low percentage of the respondents would treat gingivitis only in emergency.

\section{Specific Treatment for Chronic Periodontitis in a Pregnant Patient}

The eighth question referred to the period during pregnancy when the treatment for chronic periodontitis can be performed. There were 152 answers, of

a. Which 60 answers (39.5\%) were the first option,

b. Only 3 answers (2\%) were the second option,

c. 24 answers $(15.8 \%)$ were the third option,

d. 4 answers $(2.6 \%)$ were the fourth option and

e. $\quad 61$ answers $(40.1 \%)$ were the fifth option.

Therefore, the opposite options collected the most numerous answers. This can be explained by the fact that the question was not clear enough about the type of treatment required.

\section{Dental Extraction in the Pregnant Patient}

The ninth question referred to the period during pregnancy when the extractions can be performed. There were 159 responses,

a. Of which 107 answers (67.3\%) were the first option,

b. Only 2 answers (1.3\%) were the second option,

c. $\quad 34$ answers $(21.4 \%)$ were the third option,

d. 9 answers (5.7\%) were the fourth option, and e. $\quad 7$ answers $(4.3 \%)$ were the last option.

The first variant was chosen by most of the dentists, showing that they would postpone the extraction, until after birth. About a quarter of participating doctors would perform the extraction in the second trimester of pregnancy, and the fewest would extract teeth anytime during pregnancy.

\section{Dental x-rays in a Pregnant Patient}

In response to the tenth question, there were 135 answers, of which

a. 93 answers (68.9\%) were the first option,

b. Only 1 answer $(0.7 \%)$ was the second option,

c. 23 answers $(17 \%)$ were the third option,

d. $\quad 11$ answers (8.1\%) were the fourth option and

e. 7 answers $(5.3 \%)$ were the last option.

Most of the dentists chose the first option, showing that they do not want to be responsible for potential complications of X-rays in the fetus.

\section{Use of Anesthetic Substances in the Pregnant Patient}

In response to this question, the total of 128 answers were distributed as follows: 41 answers (32\%) were negative, and 87 answers $(68 \%)$ were affirmative. The 87 responses referring to the most frequently used anesthetic substances were divided as follows: articain (49.4\%), mepivacaine (43.7\%) and lidocaine $(6.9 \%)$ (Figure 4).

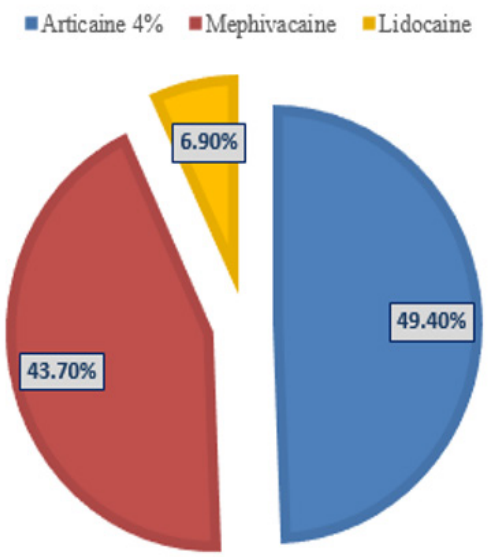

Figure 4: Graphical representation of the most frequently used anesthetic substances in pregnant patients.

\section{Prescription of Antibiotics in the Pregnant Patient}

In response to this question, the 128 answers were divided into 83 negative responses (64.8\%) and 45 affirmative responses (35.2\%). Regarding the most frequently prescribed antibiotics, the 45 responses were distributed as follows: amoxicillin (40\%), augmentin (31.1\%), prescribed only after consulting the gynecologist (15.4\%), ampicillin (8.88\%), zinnat $(2.26 \%)$, any compatible antibiotic (2.26\%) (Figure 5). 


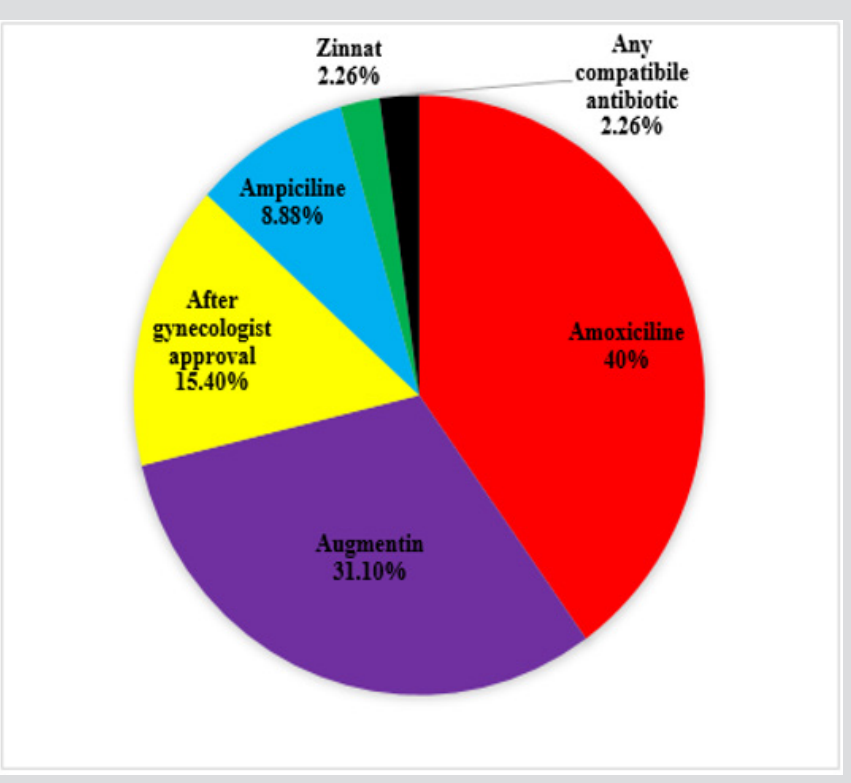

Figure 5: Graphical representation of the most frequently prescribed antibiotics in pregnant patients.

\section{Discussion}

Maintaining the dental and oral health is essential during pregnancy. Alteration of overall health and the medical interventions during pregnancy may have negative effects on the development of the fetus. Even if they are aware of the importance of maternal oral health, many dentists have a reserved attitude when in the position to provide dental care to pregnant women due to the risks involved [18]. Moreover, the lack of consensus between dentists and gynecologists, could be a significant discouragement for pregnant women seeking dental care [19]. In our study, all the dentists of different specialties (dento-alveolar surgery, periodontics, endodontics, prosthesis, orthodontics, and general dentistry), would agree to treat a pregnant patient regardless of the period of pregnancy. However, about one third of dentists would treat a pregnant woman like any other patient, thus ignoring the potential complications and risks. Dental problems challenged most of the doctors participating in our study. More than one third of the doctors would perform a dental treatment anytime during pregnancy, and about the same number of doctors were at the opposite position and would treat only serious cases.

If the patient experiences pain located at the level of a tooth affected by profound dental caries, also involving pulp pathology and an endodontic treatment is required, it should be done regardless of the stage of pregnancy. The elective treatment consists of vital pulpectomy under plexal anesthesia without vasoconstrictor [5]. As an emergency, acute pulpitis should be treated by $43 \%$ of the doctors in our study. Approximately a quarter of the doctors consider the second trimester to be the most appropriate for such treatments. For dental pulp necrosis, there are two possible therapeutic approaches: conservative treatment or extraction. The conservative treatment aims at preserving the tooth and involves several steps: removal of the necrotic and infected pulp tissue, mechanical treatment and sterilization of the root canals, root canal filling and coronary reconstruction. The tooth extraction is indicated when the pulp necrosis is complicated with periapical periodontitis and the tooth is severely affected, and the coronary reconstruction is impossible. If considered an emergency, 33.5\% of the dentists would treat this pulp pathology. Most doctors, $43.3 \%$, consider this pathology to be treated at any stage of pregnancy.

Gingivitis is an inflammation of the gingiva, often caused by the accumulation of bacterial plaque and tartar which cause irritation and induce inflammation. First-treatment plan is professional scaling and professional cleaning performed in the dental office. A percentage of $70 \%$ of the dentists would treat this gingival affliction at any stage of pregnancy and 15\% only emergency cases. Dental extraction is a challenge for the dentists, especially if the patient is pregnant. Extractions can be performed between the 4th month of pregnancy and the end of the 8th month of pregnancy (2nd trimester and partly 3rd trimester) [13]. Most practitioners (68\%) consider that this procedure should only be done in emergency situations. A very small percentage $(4 \%)$ would perform a dental extraction anytime during pregnancy. The most commonly used anesthetic substances, which, according to the responses given by the doctors, were: articaine (49\%), mepivacaine (44\%) and lidocaine $(7 \%)$, could have some side effects in pregnant patients. Once absorbed in the general circulation, these substances can penetrate the placental barrier and reach the fetus. Although the toxic effect of these anesthetics is well known, a high percentage $(68 \%)$ of the dentists would use these substances, whereas $32 \%$ would not use them in dental procedures. Although lidocaine is preferred, the preference for articaine could be explained by its wide use in current dental procedures. Of the total respondents, onethird would not perform anesthesia in a pregnant woman without considering the risk-benefit factors. According to the international 
literature, some authors recommend that no dental x-rays [13] should be performed during the first 8 months of pregnancy.

However, there are studies that have shown that exposure to radiation during dental $\mathrm{x}$-rays is very low, being harmless to a pregnant patient [17]. Physicians participating in this study do not perform dental x-rays in a proportion of $68.9 \%$. A study in the Davangere district in Karnataka, India, assessed the dentists' awareness regarding the treatment protocols in pregnant women. The conclusions were that there is a clear lack of knowledge about the proper management of pregnant women, and dentists require continuous medical education and a postgraduate curriculum for the management of such situations [13]. Another study, conducted in Brazil, assessed the current knowledge and recommendations of gynecologists and dentists regarding dental care in pregnant women. The questionnaires approached the following issues: the oral health during pregnancy, the collaboration between prenatal care providers and dental services providers, the administration of prenatal fluoride supplements, the selection of local anesthetic substances for pain control, the treatment of infections and the dental procedures which can be performed during every quarter. Most doctors only provided dental care when a dental problem was mentioned, with limited prophylaxis.

The results showed that dental management during pregnancy still had some deviations from the recommendations in the literature, indicating the need for updating the knowledge of these two categories of health professionals $[19,20]$. In France, a study conducted on 460 health professionals, 100 gynecologists and midwives, and 360 dentists, evaluated the knowledge, the approach and the daily medical practices. Their knowledge regarding the oral changes during pregnancy, the possible association of periodontal pathology with premature birth and low birth weight and behavior towards their patients were assessed. The most common oral manifestations mentioned by all the practitioners were gingival bleeding and gingivitis. All professionals have shown a lack of information on the subject. The results highlighted the need for continuous education regarding pregnancy and oral health conditions. Moreover, the update of the therapeutic approaches is essential in order to effectively prevent the risks such as preeclampsia and premature birth [21]. The risks associated to the radiation exposure of a pregnant patient often results in inadequate dental care upon an emergency, and a study including 250 dentists in Tabriz, Iran, showed that the population did not have sufficient knowledge about the risk of performing dental radiographs during pregnancy. Most radiology specialist use during dental radiography a sort lead $92 \%$ and only a few use a long rectangular collimator $3,2 \%[22]$.

\section{Conclusion}

As a general conclusion, the dentists included in our study had insufficient knowledge regarding the particularities of the dental treatments that can be performed in pregnant women. Therefore, postgraduate courses would be necessary for updating the medical education of dental practitioners.

\section{Acknowledgment}

Aranka Ilea, Adela Cristina Lazar and Adriana Emanuela Morar have equals contributions as first authors of the article.

\section{References}

1. Naseem M, Khurshid Z, Khan HA, Niazi F, Zohaib S, Zafar MS (2016) Oral health challenges in pregnant women: Recommendations for dental care professionals. The Saudi Journal for dental research (7):138-146.

2. Kumar J, Renee S (2009) Oral health care during pregnancy recomandation for oral health professionals. New York State Dental Journal 18: 29-33.

3. Singla N, Singla R (2013) Oral Health Care during pregnancy. Guident 6: 64-66.

4. Dumitru A, Păunică S, Giurgiu M, Cureu M (2009) Măriri de volum gingival, clinică și principii de tratament (Eds.), Didactică și pedagogică, București, Romania.

5. Grigore B (2002) Reabilitare Orală. Medicală Universitară Iuliu Hațieganu, Cluj-Napoca, (Eds). Romania.

6. Krauer B, Krauer F (1977) Drug kinetics in pregnancy. Clin Pharma-cokinet 2(3): 167-181.

7. Andrade S, Gurwitz J, Davis R, Chan K, Finkelstein J, Fortman K (2004) Prescription drug use in pregnancy. Obstetrics Gynecology 191(2): 398407.

8. Grahame Smith D, Aronson J (2002) Oxford textbook of clinical pharmacology and drug therapy. Oxford University Press; ( $3^{\text {rd }}$ edn.), 95(9): 472.

9. (1994) Teratology Society Public Affair Committee. FDA classification of drugs for teratogenic. Teratology pp. 446-447.

10. Bomford J, Ledger J, O`Keeffe B, Reiter C (1993) Ciprofloxacin use during pregnancy. Drugs 45: 461-462.

11. Kazy Z, Puho E, Czeizel A (2005) Teratogenic potential of vaginal metronidazole treatment during pregnancy. Journal of Obstetrics and Gynecology 123(2): 174-178.

12. Cristian M, Chiriac I, Cristian G, Vlădăreanu R, Gheorghe M (2010) Particularități ale tratamentului stomatologic în sarcină. Revista Română de Stomatologie 4(56):269-271.

13. Roman A (2001) Parodontita asociată factorilor sistemici, implicații terapeutice, Teodesco, Cluj-Napoca, (Eds.). Romania.

14. Lung T (2007) Parodontologie Clinică. Napoca Star, Cluj-Napoca, (Eds.), Romania.

15. Yamoah KK, Lindow S, Karsai L (2009) A large epulis in pregnancy. J Obstet Gynaecol 29(8): 761-762.

16. Khairallah A (2015) Dental care throughout pregnancy: Do`s and Don`ts. Smile Dental Journal 1(10): 16-20.

17. Cengiz S (2007) The pregnant patient: Consideration for dental management and drug use. Quintessence International 3(38): 133-142.

18. Shruthi P, Mohankumar K (2013) Awareness of Dental Treatment Protocol for Pregnant Women and Lactating Mother's in General Dental Practitioners of Davangere District, Karnataka, India. Journal of Clinical and Diagnostic Research 7(12): 3126

19. Ajesh G, Simin S, Johnson M (2012) How Do Dental and Prenatal Care Practitioners Perceive Dental Care During Pregnancy? Current Evidence and Implications. Birth 39(3): 238-247.

20. Zanata L, Barros Parnon K, Navarro Lopes P (2008) Prenatal dental care: evaluation of professional knowledge of obstetricians and dentists in the cities of Londrina/PR and Bauru/SP, Brazil. Journal of Applied Oral Science 16(3): 194-200 
21. Boutigny H, de Moegen M, Egea L, Badran Z, Boschin F, et al. (2016) Oral Infections and Pregnancy: Knowledge of Gynecologists/Obstetricians, Midwives and Dentists. Oral Health and Preventive Dentistry 14(1): 4147.

\section{ISSN: 2574-1241}

DOI: 10.26717/BJSTR.2019.15.002667

Lecturer Bianca Adina Boșca. Biomed J Sci \& Tech Res

(C) This work is licensed under Creative

Submission Link: https://biomedres.us/submit-manuscript.php
22. Tahmineh R, Bazvand R, Ghojazadeh M (2011) Diagnostic Dental Radiation Risk during Pregnancy: Awareness among General Dentists in Tabriz. Dental Research, Dental Clinics, Dental Prospects 5(2): 67-70.

$\begin{array}{ll}\text { BIOMEDICAL } & \text { Assets of Publishing with us } \\ \text { RESEARCHES } & \text { - Global archiving of articles } \\ & \text { - Immediate, unrestricted online access } \\ \end{array}$

